\title{
Intermolecular interactions and charge transfer in the 2:1 tetrathiafulvalene bromanil complex, $(\text { TTF })_{2}$-BA
}

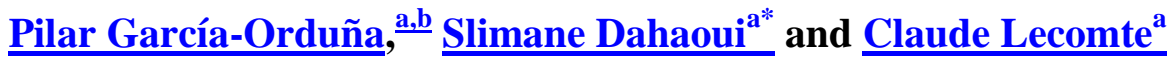 \\ ${ }^{a}$ Laboratoire de Cristallographie, Résonance Magnétique et Modélizations (CRM2) \\ UMR CNRS-UHP 7036, Institut Jean Barriol, Université Henri Poincaré, BP \\ 70239, 54506 Vandoeuvre-les-Nancy, France, and ${ }^{\mathbf{b}}$ Departamento de Química \\ Inorgánica, Instituto de Síntesis Química y Catálisis Homogénea (ISQCH), \\ Universidad de Zaragoza CSIC, 50009 Zaragoza, Spain \\ Correspondence e-mail: slimane.dahaoui@crm2.uhp-nancy.fr
}

The crystal structure of the 2:1 charge-transfer complex of tetrathiafulvalene [2,2'-bis(1,3-dithiolylidene)] and bromanil (tetrabromo-1,4-benzoquinone) [(TTF $\left.)_{2}-\mathrm{BA},\left(\mathrm{C}_{6} \mathrm{H}_{4} \mathrm{~S}_{4}\right)_{2}-\mathrm{C}_{6} \mathrm{Br}_{4} \mathrm{O}_{2}\right]$ has been determined by $\mathrm{X}$-ray diffraction at room temperature, 100 and $25 \mathrm{~K}$. No structural phase transition occurs in the temperature range studied. The crystal is made of TTF-BA-TTF sandwich trimers. A charge-transfer estimation between donor and acceptor $(0.2 \mathrm{e})$ molecules is proposed in comparison to the molecular geometries of TTF-BA and TTF and BA isolated molecules. Displacement parameters of the molecules have been modeled with the TLS formalism.

Keywords: tetrathiafulvalene; bromanil; thermal motion analysis; supramolecular chemistry; charge-transfer complex; intermolecular interactions..

\section{Introduction}

Understanding strong and weak interatomic interactions enables the design and manipulation of molecular systems whose physical properties depend on crystal packing. The importance of such a study is shown by many examples, such as organic chargetransfer (CT) complexes which are built by co-crystallization of organic planar electron donor $(D)$ and acceptor $(A)$ molecules, often aromatic rings. The properties of these complexes depend on their crystal packing which drives the interactions between $D$ and $A$ to control the charge transfer. Most studies concern 1:1 charge-transfer complexes in which

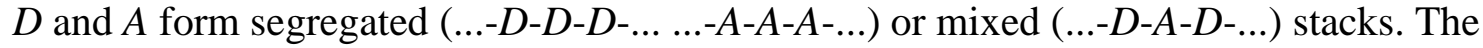
former generally present a high electric conductivity in the direction of stacking (Cohen et al., 1974 $\Rightarrow$ ). The latter are usually insulators or semiconductors at ambient conditions. Some of them undergo an unusual phase transition, called a neutral-ionic phase transition, related to the variation of the partial degree of charge transfer $\left(q_{\mathrm{TC}}\right.$; Torrance \& Mayerle, 
$1981 \Rightarrow$; Le Cointe et al., $1995 \Rightarrow$; García et al., 2007 $\Rightarrow$ ). This phase transition is accompanied by structural modifications of the stacking, most often with symmetry breaking, with the formation of $D$-A pairs. We have recently shown the possibility of characterizing the two microscopic control parameters (dimerization and charge transfer) via experimental charge-density studies (García et al., 2007 $\Rightarrow$ ).

By applying temperature, pressure and light excitation charge transfer may also be induced in 2:1 molecular complexes, but no structural evidence has been published yet. Previous studies concerned pressure evolution of the ionicity of the $D$ and $A$ molecules from spectroscopic studies: it has been proposed that ionicity increases with pressure (Matsuzaki et al., 1992 $\Rightarrow$; Tasaki et al., 1997 $\Rightarrow$ ), and a non-uniform charge distribution between two moieties of the $D-A-D$ trimer was deduced from electron-molecular vibration coupling (Matsuzaki \& Yartsev, 1994 $\Rightarrow$; Basaki et al., 1997 $\Rightarrow$ ). Moreover, this non-uniform charge distribution has also been observed on $A$ sites, suggesting that coupled trimers may behave cooperatively (Sadohara \& Matsuzaki, 1997 $\Rightarrow$ ).

Therefore, it is essential to characterize the intermolecular interactions which occur in these complexes. In the present study we analyzed the evolution of the crystal structure of the 2:1 charge-transfer complex of tetrathiafulvalene and bromanil [(TTF $)_{2}-\mathrm{BA},\left(\mathrm{C}_{6} \mathrm{H}_{4} \mathrm{~S}_{4}\right)_{2}-$ $\mathrm{C}_{6} \mathrm{Br}_{4} \mathrm{O}_{2}$ ] from $25 \mathrm{~K}$ to room temperature as measured by accurate single-crystal X-ray diffraction. Donor (TTF) and acceptor (BA) molecules are represented below.<smiles>C1=CSC(=C2SC=CS2)S1</smiles><smiles>O=C1C(Br)=C(Br)C(=O)C(Br)=C1Br</smiles>

\section{Experimental}

\subsection{TTF $_{2}$-BA crystallization}

TTF and BA powders were purchased from Lancaster and purified. Single crystals of $(\mathrm{TTF})_{2}$-BA were grown by sublimation of the component materials in a vacuum-sealed Pyrex glass tube, which was placed in a specially designed homemade electrical furnace, with two coils. This allows the two compounds to be heated at two different temperatures, $T_{1}=388 \mathrm{~K}$ and $T_{2}=352 \mathrm{~K}$, which are the sublimation temperatures of BA and TTF, respectively. After 2 weeks dark prismatic-like crystals were obtained.

\subsection{X-ray crystallography}

The temperature evolution of the unit-cell parameters between 100 and $293 \mathrm{~K}$ was studied by single-crystal diffraction on an Xcalibur-Saphire2 CCD Oxford Diffraction diffractometer, with Mo $K a$ radiation. The crystal was cooled using an Oxford Cryostream $\mathrm{N}_{2}$ open-flow cryostat. The unit-cell parameters were determined from the analysis of diffracted intensities on the same 20 images, with a fixed detector position and four 
different values for $\varphi$. The unit-cell parameter evolution is given in Fig. S1 (supplementary material $^{\underline{1}}$ ) and does not show any structural phase transition.

The crystal structure X-ray diffraction data were collected at 293, 100 and $25 \mathrm{~K}$. An Oxford-Helijet open-flow He gas cryostat was used for the $25 \mathrm{~K}$ experiment. Images were collected at a fixed detector position using $115^{\circ}$ wstep scans repeated at 4 different $\varphi$ angle values. The 100 and $25 \mathrm{~K}$ diffraction data were collected for a charge-density analysis which explains the number of measured data; however, their quality was not good enough for a physically meaningful electron-density model. The lower number of data collected at $25 \mathrm{~K}$ accounts for geometrical limitations due to the He blower during data collection.

Data processing was performed using the CrysAlis Red program (Oxford Diffraction Ltd, $2010 \Rightarrow$ ). Absorption was corrected for by numerical methods based on crystal-faces indexing with $A B S O R B$ (DeTitta, $1985 \Rightarrow$ ). Such a correction was deemed necessary to obtain reliable data $\left(T_{\min }=0.086, T_{\max }=0.282\right.$, see Table $\left.1 \Rightarrow\right)$; it may be surprising that $T_{\min }$ and $T_{\max }$ are different from one experiment to another, but it is in line with the number of reflections collected. In the room-temperature study the total number of collected data is much smaller and the angular conditions of the reciprocal lattice nodes to enter the Ewald sphere are not the same, and consequently the incoming and diffraction optical pathways depend on the experiment. Reflections having $I>2 \sigma(I)$ were used. The structures were solved by direct methods (Sheldrick, 2008 $\Rightarrow$ ) and successive Fourier synthesis, and then refined by full-matrix least-square refinements against $F^{2}$ using the SHELXL97 program (Sheldrick, 2008 $\Rightarrow$ ). All non-H atoms were refined anisotropically. $\mathrm{H}$ atoms were observed in Fourier maps, then refined riding on their attached atoms with isotropic displacement parameters fixed at 1.2 times the $U_{\text {eq }}$ of the attached atoms. The type of space group does not change and no discontinuity is observed on the metric of the unit cell, from 293 to $25 \mathrm{~K}$, confirming that no phase transition occurs, contrary to TTF-CA [tetrathiafulvalene chloranil (tetrachloro-1,4-benzoquinone) complex]. The asymmetric unit contains one TTF molecule and half a bromanil, which lies on the $\left(\begin{array}{lll}1 / 2 & 0 & 0\end{array}\right)$ inversion center of the $P 2_{1} / n$ space group. All atoms are on general positions. The final positions and displacement parameters are given in the supplementary material together with all the geometrical parameters. Further details on the crystal data and experimental conditions are given in Table $1 \Rightarrow$.

\section{Table 1}

Experimental details

For all structures: $2 \mathrm{C}_{6} \mathrm{H}_{4} \mathrm{~S}_{4} \cdot \mathrm{C}_{6} \mathrm{Br}_{4} \mathrm{O}_{2}, M_{\mathrm{r}}=416.20$, monoclinic, $P 2_{1} / n, Z=4$. Experiments were carried out with Mo $K a$ radiation using an Xcalibur-Saphire2 diffractometer. Absorption: integration using $A B S O R B$ (DeTitta, $1985 \Rightarrow$ ). Refinement was on 145 parameters with 0 restraints. H-atom parameters were not refined.

$293 \mathrm{~K} \quad 100 \mathrm{~K} \quad 25 \mathrm{~K}$

Crystal data

$a, b, c(\AA)$

10.3653 (7), 11.7998

(7), 11.0749 (7)

10.2781 (9), 11.5982

(8), 11.0418 (9)

10.2507 (6), 11.5276

$\beta\left({ }^{\circ}\right)$

110.217 (6)

110.434 (8)

(9), $10.9799(9)$

$V\left(\AA^{3}\right)$

$1271.10(14)$

$1233.44(17)$

$110.446(7)$

$1215.71(15)$ 


\begin{tabular}{llll}
\hline$\mu\left(\mathrm{mm}^{-1}\right)$ & 7.01 & 7.22 & 7.33 \\
Crystal size $(\mathrm{mm})$ & $0.40 \times 0.40 \times 0.20$ & $0.40 \times 0.40 \times 0.20$ & $0.40 \times 0.40 \times 0.20$ \\
& & & \\
Data collection & & & $0.009,0.233$ \\
$T_{\min }, T_{\max }$ & $0.113,0.236$ & $0.086,0.282$ & $48219,3936,3775$ \\
$\begin{array}{l}\text { No. of measured, independent and } \\
\text { observed }[I>2 \sigma(I)] \text { reflections }\end{array}$ & $18082,3761,2563$ & $51302,3972,3905$ & \\
$(\sin \theta / \lambda)_{\max }\left(\AA^{-1}\right)$ & 0.727 & 0.727 & 0.727 \\
Completeness $(\%)$ & 92 & 99 & 99 \\
$R\left[F^{2}>2 \sigma\left(F^{2}\right)\right], w R\left(F^{2}\right), S$ & $0.038,0.105,0.99$ & $0.033,0.083,1.12$ & $0.025,0.067,1.10$ \\
No. of reflections & 3761 & 3761 & 3936 \\
$\Delta \rho_{\max }, \Delta \rho_{\min }\left(\mathrm{e} \AA^{-3}\right)$ & $0.71,-0.68$ & $1.31,-0.70$ & $0.96,-0.82$
\end{tabular}

Computer programs used: KappaCCD (Nonius, $1998 \Rightarrow$ ), DENZO and SCALEPAK (Otwinowski \& Minor, $1997 \Rightarrow$ ), CrysAlis (Oxford Diffraction Ltd, 2010 $\Rightarrow$ ), SHELXS97, SHELXL97 (Sheldrick, $2008 \Rightarrow$ ), ORTEPIII (Farrugia, $1997 \Rightarrow$ ), Win GX publication routines (Farrugia, 1999 $\Rightarrow$ ).

\section{Results and discussion}

No mixed or segregated stack is observed in (TTF $)_{2}$-BA in contrast to the $1: 1$ complex (García et al., 2005 $\Rightarrow$ ); in this crystal structure the building block is a trimer. The packing mode is more similar to the sandwich herringbone structure defined by Gavezzotti \& Desiraju $(1988 \Rightarrow$ ) and Desiraju \& Gavezzotti $(1989 \Rightarrow$ ) for aromatic hydrocarbons than to the typical arrangement of charge-transfer complexes, where $D$ and $A$ form mixed or segregated stacks. In (TTF $)_{2}$-BA the special disposition in orthogonal trimers produces layers, almost parallel to the (101) plane, as shown in Fig. $1 \Rightarrow$. Thus, in these layers each bromanil molecule is surrounded by four TTF molecules: two TTF molecules of the trimer and two others belonging to two orthogonal neighboring trimers. The resulting motif is a cage centered on the BA molecule (Fig. $2 \Rightarrow$ ).

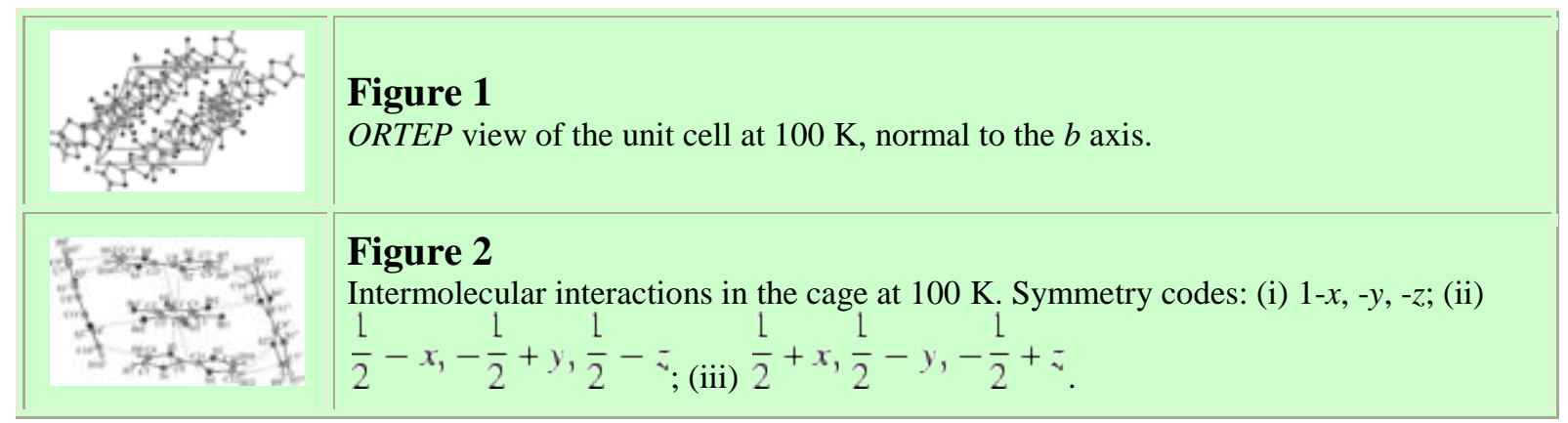

The relative disposition of the donor and acceptor molecules of the trimer is characteristic of TTF and benzoquinone charge-transfer complexes (Frankenbach et al., 1991 $\Rightarrow$ ) or of complexes substituted benzoquinones like the chloranil (Mayerle \& Torrance, $1981 \Rightarrow$ ), bromanil (García et al., $2005 \Rightarrow$ ) and fluoranil (Mayerle \& Torrance, $1981 \Rightarrow$ ). The longest axes of each molecule are rotated out of alignment. This rotation between donor and acceptor molecules allows stabilization to be achieved in the overlap of the HOMO orbital 
of the donor and the LUMO orbital of the acceptor (overlap that would be forbidden as these orbitals have opposite symmetry; Mayerle \& Torrance, 1981 $\Rightarrow$ ). In 1:1 TTF-AA charge-transfer complexes ( $X$ being the halogen atom) the angle between the $\mathrm{O}_{\ldots}, \mathrm{O}$ axis tends to be orthogonal to the longer axis of the TTF molecule when the $\mathrm{C}-X / \mathrm{C}=\mathrm{O}$ ratio of bond lengths increases. However, when comparing TTF-BA and (TTF) $)_{2}-\mathrm{BA}$, even if this ratio is equal (1.53) for both complexes the angle between the TTF axis and the $\mathrm{O}_{\ldots}, \mathrm{O}$ direction in (TTF) $)_{2}$-BA $\left[111.6(2)^{\circ}\right]$ is not as close to $90^{\circ}$ as those observed for TTF-BA [90.0 (5) and $\left.93.8(5)^{\circ}\right]$. We can thus conclude that the overlap between HOMO and LUMO orbitals is not optimal in the trimer. The ground state of the 2:1 complex is thus expected to be less ionic than for TTF-BA $\left(q_{\mathrm{CT}} \simeq 0.9 \mathrm{e}\right.$; Girlando et al., $1985 \Rightarrow$; García et al., $2005 \Rightarrow$ ). This can be geometrically verified by comparing the crystal structures of TTF and BA (in each case neutral molecules) with those in 1:1 and 2:1 complexes. Analogous to chloranil (Mayerle \& Torrance, $1981 \Rightarrow$ ), the simple C-C bonds of bromanil molecules are related to the lowest unoccupied orbital (LUMO) which is bonding with respect to these bonds, and antibonding with respect to the double $\mathrm{C}=\mathrm{C}$ and $\mathrm{C}=\mathrm{O}$ bonds. Thus, when the molecular charge increases the former should be shorter while the latter should be longer. This tendency is clearly seen when comparing these bond lengths in BA, TTF-BA and $(\mathrm{TTF})_{2}$-BA crystal structures at the same temperature $(100 \mathrm{~K})$. Simple C-C bonds in (TTF) $)_{2}$-BA have values of 1.493 (3) $\AA$, equal (within a standard deviation) to 1.491 (2) $\AA$ found in the bromanil molecule in its BA crystal (García-Orduña et al., 2011 $\Rightarrow$ ), opposite to those found in TTF-BA, which means that the $\mathrm{C}-\mathrm{C}$ bond length is 1.458 (5) $\AA$. The double $\mathrm{C}=\mathrm{C}$ and $\mathrm{C}=\mathrm{O}$ bonds behave in the same way with almost equal lengths in $\mathrm{BA}$ and $(\mathrm{TTF})_{2}$-BA crystals (Table $2 \Rightarrow$ ). The geometric parameters of the TTF molecule are more sensitive to the charge transfer and were used to estimate the $q_{\mathrm{CT}}$ charge transfer in the 1:1 complexes (Umland et al., $1988 \Rightarrow$ ). In the case of the 2:1 charge-transfer complexes, where the charge transfer may involve both TTF molecules, deviation from neutral geometry is expected to be smaller: the central $\mathrm{C}=\mathrm{C}$ bond length $[1.354(4) \AA$ at $100 \mathrm{~K}]$ is slightly longer than the corresponding one in the TTF crystal [1.340 (4) A], and much shorter than those in TTF-BA [1.400 (4) and 1.397 (4) $\AA$ ] . This small variation of the $\mathrm{C}=\mathrm{C}$ bond length may be related to the partial charge transfer $\left(q_{\mathrm{CT}}\right)$ in the complex. The HOMO of TTF is $\pi$-bonding with respect to this bond, thus when $q_{\mathrm{CT}}$ increases the $\mathrm{C}=\mathrm{C}$ bond length should increase (Katan, 1999 $\Rightarrow$ ). An approximate charge transfer of 0.2 e can then be evaluated from both the ratio $(r / s)$ and the difference $(r-s)$ of TTF geometric parameters for $(\mathrm{TTF})_{2}-\mathrm{BA}$ in the whole temperature range studied (see Umland et al., $1988 \Rightarrow$, for definitions of the $r$ and $s$ parameters). Hence, the central $\mathrm{C}=\mathrm{C}$ bond length of the TTF molecule in the trimer does not change with temperature [1.354 (4), 1.360 (3) and 1.360 (3) $\AA$ at 293, 100 and $25 \mathrm{~K}$, respectively]. Thus, based on this criteria used by all authors when no charge-density study is available, the molecules seem to be partially charged between $25 \mathrm{~K}$ and room temperature, and the resulting complex may be written as $\left(\mathrm{TTF}^{0.2+}\right)_{2} \mathrm{BA}^{0.4-}$. This assumption is in line with the non-planarity of the TTF molecule. Deviation of the non-H atoms from the mean plane of the molecule for the three studied temperatures is reported in the supplementary material . TTF is a very flexible molecule whose conformation depends on the intermolecular interactions in the crystal. At room temperature the largest displacement from the mean plane defined by the non-H atoms [0.060 (3) $\AA]$ is observed for the central C8 atom. This value increases to 0.085 (2) and 0.0915 (2) $\AA$ at 100 and $25 \mathrm{~K}$. In the TTF-BA structure all non-H atoms of TTF are 
coplanar within \pm 0.012 (1) and \pm 0.028 (1) $\AA$ in high- and low-temperature phases. In the trimer the bending angle (i.e. the dihedral angle between each cycle of the molecule) is $4.9(1)^{\circ}$ at room temperature and reaches $6.8(1)$ and $7.3(1)^{\circ}$ at 100 and $25 \mathrm{~K}$. The torsion angles around the central $\mathrm{C}=\mathrm{C}$ bond also reflect the distortion of the molecule: $\mathrm{S} 2-\mathrm{C} 8-\mathrm{C} 11-$ $\mathrm{S} 3$ and S4-C8-C11-C1 are not similar [-2.0 (4) and $\left.-0.7(4)^{\circ}\right]$ at room temperature, and the difference is larger at $25 \mathrm{~K}\left[-3.5(2)\right.$ and $\left.0.0(2)^{\circ}\right]$. These geometrical parameters indicate that the TTF molecule is more distorted than those observed in the TTF-BA dimer units.

\begin{tabular}{|c|c|c|c|c|c|}
\hline \multicolumn{6}{|c|}{ 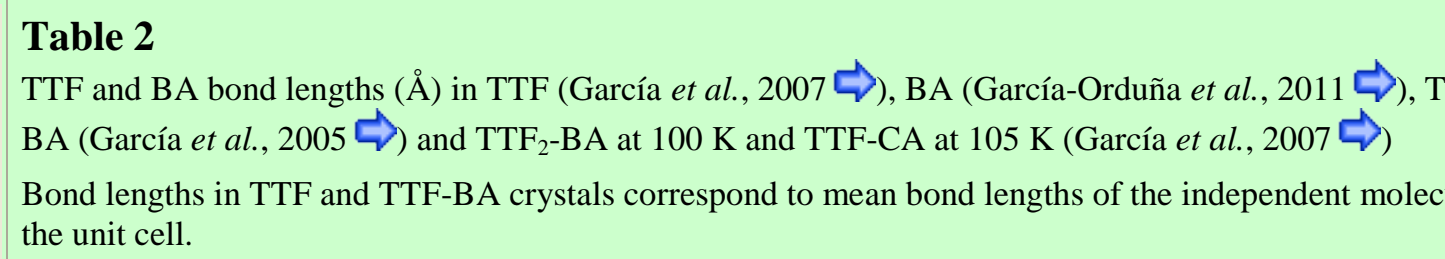 } \\
\hline & TTF & $\mathbf{B A}$ & TTF-CA & TTF-BA & $(\mathrm{TTF})_{2}-\mathrm{BA}$ \\
\hline $\mathrm{C}=\mathrm{C}_{\text {central }}$ & $1.340(4)$ & - & $1.3678(7)$ & $1.398(4)$ & $1.360(3)$ \\
\hline C-S $S_{\text {central }}$ & $1.765(7)$ & - & $1.7497(4)$ & $1.725(3)$ & $1.760(4)$ \\
\hline C-S & $1.752(5)$ & - & $1.7423(5)$ & $1.729(3)$ & $1.746(4)$ \\
\hline $\mathrm{C}=\mathrm{C}$ & $1.322(3)$ & - & $1.7500(4)$ & $1.338(4)$ & $1.346(4)$ \\
\hline $\mathrm{C}=\mathrm{C}$ & - & $1.343(2)$ & - & $1.462(4)$ & $1.358(3)$ \\
\hline $\mathrm{C}-\mathrm{C}$ & - & $1.491(2)$ & - & $1.371(3)$ & $1.493(3)$ \\
\hline $\mathrm{C}=\mathrm{O}$ & - & $1.217(2)$ & - & $1.241(4)$ & $1.229(3)$ \\
\hline $\mathrm{C}-\mathrm{Br}$ & - & $1.8615(15)$ & - & $1.887(3)$ & 1.878 \\
\hline
\end{tabular}

Therefore, when (TTF) $)_{2}$-BA is formed the major geometrical changes concern the TTF molecule, contrary to TTF-BA and TTF-CA complexes; it leads to an estimated charge transfer of $q_{\mathrm{CT}}=0.2 \mathrm{e}$ from each TTF, compared with $0.9 \mathrm{e}$ in TTF-BA and 0.74 (2) e in the antiferroelectric TTF-CA phase (García et al., 2007 $\Rightarrow$ ). The resulting estimated BA charge (0.4 e) does not seem to strongly affect its geometry. Furthermore, joint experimental and theoretical accurate charge-density studies of TTF-BA and (TTF) $)_{2}$-BA followed by a Bader partitioning (Bader \& Essén, 1984 $\Rightarrow$ ) should give a definitive answer about $q_{\mathrm{CT}}$ and its relation to the geometry of $X \mathrm{~A}$ molecules.

Several other geometrical features point to the strong connection between donor and acceptor molecules in the trimer. Donor and acceptor molecules are not parallel, their mean planes form a dihedral angle of $3.20(8)^{\circ}$, very close to that observed for TTF-CA $3.0^{\circ}$ and smaller than those found in TTF-FA, $4.2^{\circ}$ (Mayerle et al., 1979 $\Rightarrow$ ), and TTF-BA, 5.5 (5) and $4.1(6)^{\circ}$ (García et al., 2005 $\Rightarrow$ ). When cooling, this dihedral angle remains constant but molecules become closer in line with the thermal contraction of the unit cell. The dimensions of the cage (Fig. $2 \Rightarrow$ ), estimated from the distance between the TTF and BA molecule centers, decrease from $7.38(3) \times 13.78(3) \AA^{2}$ at room temperature to $7.23(3) \times$ $13.69(3) \AA^{2}$ and $7.18(3) \times 13.64(3) \AA^{2}$ at 100 and $25 \mathrm{~K}$.

Intermolecular interactions in the cage are shown in Fig. $2 \Rightarrow$ and their geometrical parameters are summarized in Table $3 \Rightarrow$. The stronger contact in the trimer takes place 
between the $\pi$-electrons of the benzene ring and the C8-S1 bond of the TTF molecule. In the stacking direction and between orthogonal trimers in the layer, intermolecular interactions between bromine and sulfur occur: at room temperature the $\mathrm{Br} 2 \ldots \mathrm{S} 4{ }^{\mathrm{iii}}$ [symmetry code (iii) $\frac{1}{2}+x, \frac{1}{2}-y,-\frac{1}{2}+z$ ] distance is 3.544 (4) $\AA$, shorter than the intratrimer Br2 .. S4 distance, 3.753 (4) $\AA$, and remarkably shorter than the sum of van der Waals radii $(3.80 \AA)$ ). All these contacts (not very common in the literature) depend on temperature, the distances decreasing from 3.544 (4) and 3.753 (4) to 3.486 (4) and 3.636 (4) $\AA$ when cooling from 293 to $25 \mathrm{~K}$. Thus, the bromanil molecule is trapped between the four TTF molecules.

\begin{tabular}{|c|c|c|c|}
\hline \multicolumn{4}{|c|}{$\begin{array}{l}\text { Table } 3 \\
\text { Intermolecular interactions }(\AA) \text { in the }(\mathrm{TTF})_{2} \text {-BA crystal }\end{array}$} \\
\hline & $293 \mathrm{~K}$ & $100 \mathrm{~K}$ & $25 \mathrm{~K}$ \\
\hline \multicolumn{4}{|l|}{ Interactions in the layer } \\
\hline \multicolumn{4}{|l|}{ Interactions in the trimer } \\
\hline C8-S1 . . centroid(BA) & $3.385(4)$ & $3.290(4)$ & $3.260(4)$ \\
\hline $\mathrm{Br} 2 \ldots \mathrm{S} 4$ & $3.753(4)$ & $3.662(3)$ & $3.636(4)$ \\
\hline \multicolumn{4}{|l|}{ Between the trimers } \\
\hline $\mathrm{Br} 2 \ldots \mathrm{S} 4^{\mathrm{iii}}$ & $3.544(4)$ & $3.504(4)$ & $3.486(4)$ \\
\hline \multicolumn{4}{|c|}{ Interactions between the layers } \\
\hline $\mathrm{S} 1^{\mathrm{i}} \ldots \mathrm{N} 2^{\mathrm{iv}}$ & $3.298(4)$ & $3.246(3)$ & $3.223(4)$ \\
\hline $\mathrm{S} 4 \ldots \mathrm{C} 10^{\mathrm{v}}$ & $3.609(4)$ & $3.514(3)$ & $3.495(3)$ \\
\hline
\end{tabular}

Besides all these interactions in the (010) plane the supramolecular architecture of the complex is based on hydrogen bonds and van der Waals interactions between $\mathrm{S}$ atoms of TTF molecules belonging to other layers, as seen in Fig. $3 \Rightarrow$. Intermolecular interactions are given in the supplementary material $. S_{\ldots}, S$ contacts are characteristic of TTF derivatives; hence it has been considered to be an important structural element that facilitates electrical conductivity (Wudl et al., 1972 $\Rightarrow$; Ferraris et al., $1973 \Rightarrow$; Bryce, 1991 $\Rightarrow$; Yamashita et al., $1996 \Rightarrow$ ). At room temperature the $\mathrm{S} 1^{\mathrm{i}}{ }_{\mathrm{n}} . \mathrm{S} 2^{\mathrm{iv}}$ distance is 3.298 (4) $\AA$, remarkably shorter than the sum of van der Waals radii $(3.60 \AA)$, and similar to that observed in the mixed-stack bis(ethylenedithio)tetrathiafulvalene 2,5-

dimethyltetracyanoquinodimethane (BEDTTF-Me $\mathrm{MCNQ}_{2} \mathrm{C}_{10} \mathrm{H}_{8} \mathrm{~S}_{8}-\mathrm{C}_{14} \mathrm{H}_{8} \mathrm{~N}_{4}$ ), $3.299 \AA$. This intercolumnar interaction in BEDTTF- $\mathrm{Me}_{2}$ TCNQ is comparable to those between $D$ and $A$ molecules along the mixed stack, as pointed out by Hasegawa et al. $(2000 \Rightarrow$ ). In $(\mathrm{TTF})_{2}$-BA this $\mathrm{S} \ldots \mathrm{S}$ contact is mainly along the [101] direction, and therefore the thermal contraction in this direction is not favorable. This result agrees with the anisotropy observed in the principal linear thermal expansion/contraction coefficients (see Fig. S1, supplementary material $): a_{b}\left(10.1 \times 10^{-5} \mathrm{~K}^{-1}\right)$ being much larger than the other thermal coefficients, $a_{\mathrm{a}}$ and $a_{\mathrm{c}}, 5.7$ and $1.8 \times 10^{-5} \mathrm{~K}^{-1}$. This large coefficient $a_{\mathrm{b}}$ is related to the interactions along the stack and may explain the distortion of the TTF planarity, as already discussed. 


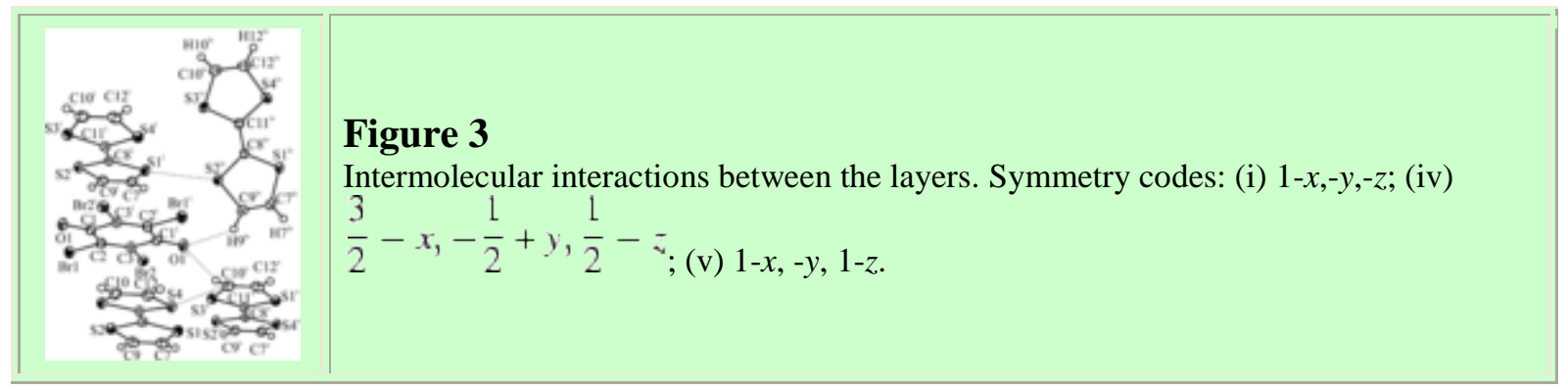

Results of the thermal motion analysis (Dunitz et al., $1988 \Rightarrow$; Schomaker \& Trueblood, $1998 \Rightarrow$ ) of the bromanil molecule in the complex, in relation to the inertial system axis, are summarized in Table $4 \Rightarrow$. For clarity, only the $\mathbf{T}$ and $\mathbf{L}$ tensors at $100 \mathrm{~K}$ are reported. $\mathbf{T}$ and $\mathbf{L}$ tensors at room temperature and $25 \mathrm{~K}$, as well as those of the TTF molecule are given in the supplementary material. At $100 \mathrm{~K}$ the $\mathbf{T}$ tensors of TTF and BA molecules are almost isotropic $\left(0.0160 \pm 0.0006 \AA^{2}\right)$. The bromanil molecule is trapped and coupled to four TTF molecules, as discussed before. The occurrence of a phase transition involving the loss of the bromanil inversion center would imply the displacement of the molecule in a specific direction out of the layer which cannot be foreseen from this TLS model. This may explain the stability of the studied phase versus $T$.

\section{Table 4}

$\mathbf{T}$ and $\mathbf{L}$ tensors of the bromanil molecule in $(\mathrm{TTF})_{2}$-BA at $100 \mathrm{~K}$, referred to as the inertial system axis

$$
\begin{aligned}
& \text { T }\left(\times 10^{-4} \check{A}^{2}\right) \\
& \left(\begin{array}{ccc}
158(3) & 3(3) & -14(4) \\
& 153(2) & 3(3) \\
& & 166(5)
\end{array}\right) \\
& \text { L ( } \left.\text { deg }^{2}\right) \\
& \left(\begin{array}{ccc}
4.7(4) & 1.1(1) & -0.5(3) \\
& 1.3(2) & -0.1(2) \\
& & 2.1(2)
\end{array}\right)
\end{aligned}
$$

Finally, if the TLS parameters of TTF and BA molecules in their crystal structures are compared with those of the 1:1 and 2:1 complexes, we note that:

- (i) translation is always isotropic;

- (ii) the principal axes of libration of the molecules do not change when forming the charge-transfer complexes;

- (iii) the main difference is the amplitude of the BA libration in the trimer $\left(4.8 \mathrm{deg}^{2}\right.$, at $100 \mathrm{~K}$ ) which is much lower than that in TTF-BA where BA molecules have a principal libration of 14.8 and $12.8 \mathrm{deg}^{2}$.

\section{Conclusions}

The structures of the 2:1 charge-transfer complex (TTF) $)_{2}$-BA at room temperature, 100 and $25 \mathrm{~K}$ have been reported. TTF and BA molecules form a herringbone packing, with orthogonal trimer units yielding layers parallel to the (101) plane. Thermal displacement analysis has been performed. Comparison with those of the isolated molecules and the 1:1 
charge-transfer complex at $100 \mathrm{~K}$ revealed that libration is smaller in the 2:1 complex, where translation of TTF and BA molecules is isotropic and quite similar. Consequently, the donor and acceptor are strongly coupled. This observation agrees with the molecular geometry, where the distortion of the TTF molecule has been shown. The TTF bond lengths indicate the existence of a partial charge transfer of 0.2 e that remains constant with temperature. On the contrary, the BA geometry suggests that the molecule remains neutral in the complex. Therefore, an accurate charge estimation is needed by using DFT calculations and high-energy synchrotron data for precise experimental density studies followed by a Bader topological analysis.

Thermal contraction enhances the $\mathrm{Br}_{n} . . \mathrm{S}$ interactions inside and between the trimers, in a very close-packing mode. In the [101] direction the crystal cohesion is held in place by remarkably short $S_{n}, S$ contacts. These close contacts prevent the possibility of the existence of a phase transition with symmetry breaking.

\section{Acknowledgements}

The authors thank the Service Commun de Diffraction X sur Monocristaux (Universite Henri Poincaré, Nancy 1) for providing access to crystallographic experimental facilities. We would also cordially thank A. Bouché for the crystal synthesis. Authors are indebted to Oxford Diffraction for lending for free the Oxford-Helijet cryostat used for the $25 \mathrm{~K}$ diffraction measurements. PGO acknowledges financial support from the CSIC, 'JAE-Doc' program, contract co-funded by the ESF.

\section{References}

Bader, R. F. W. \& Essén, H. (1984). J. Chem. Phys. 80, 1943-1960. CrossRef CAS

Basaki, S., Matsuzaki, S. \& Yartsev, V. (1997). Phys. B, 239, 171-175. CrossRef CAS Bryce, M. R. (1991). Chem. Soc. Rev. 20, 355-390. CrossRef CAS

Cohen, M. J., Coleman, L. M., Saito, A. F. \& Heeger, A. J. (1974). Phys. Rev. B, 10, 12981307. CrossRef CAS

Desiraju, G. R. \& Gavezzotti, A. (1989). J. Chem. Soc. Chem. Commun. 10, 621623. CrossRef

DeTitta, G. T. (1985). J. Appl. Cryst. 18, 75-79. CrossRef CAS Web of Sciences details

Dunitz, J. D., Maverick, E. F. \& Trueblood, K. N. (1988). Angew. Chem. Int. Ed. Engl. 27, 880-895. CrossRef

Farrugia, L. J. (1997). J. Appl. Cryst. 30, 565. CrossRef details

Farrugia, L. J. (1999). J. Appl. Cryst. 32, 837-838. CrossRef CAS details

Ferraris, J., Cowan, D. O., Walatka, V. V. \& Perlstein, J. H. (1973). J. Am. Chem. Soc. 95, 948-949. CrossRef CAS

Frankenbach, G. M., Beno, M. A. \& Williams, J. M. (1991). Acta Cryst. C47, 762-

764. CrossRef details

García, P., Dahaoui, S., Fertey, P., Wenger, E. \& Lecomte, C. (2005). Phys. Rev. B, 72, 104115.

García, P., Dahaoui, S., Katan, C., Souhassou, M. \& Lecomte, C. (2007). Faraday Discuss. 
135, 217-235. Web of Sciences PubMed

García-Orduña, P., Dahaoui, S. \& Morgenroth, W. (2011). Submitted.

Gavezzotti, A. \& Desiraju, G. R. (1988). Acta Cryst. B44, 427-434. CrossRef details

Girlando, A., Pecile, C. \& Torrance, J. B. (1985). Solid State Commun. 54, 753-

759. CrossRef CAS

Hasegawa, T., Mochida, T., Kondo, R., Kagoshima, S., Iwasa, Y., Akutagawa, T.,

Nakamura, T. \& Saito, G. (2000). Phys. Rev. B, 62, 10059-10066. CrossRef CAS

Katan, C. (1999). J. Phys. Chem. A, 103, 1407-1413. CrossRef CAS

Le Cointe, M., Lemée-Cailleau, M. H., Cailleau, H., Toudic, B., Toupet, L., Heger, G., Moussa, F., Schweiss, P., Kraft, K. H. \& Karl, N. (1995). Phys. Rev. B, 51, 33743386. CrossRef CAS

Matsuzaki, S., Hiejima, T. \& Sano, M. (1992). Solid State Commun. 82, 301-304. CAS

Matsuzaki, S. \& Yartsev, V. (1994). Solid State Commun. 89, 941-944. CAS

Mayerle, J. J. \& Torrance, J. B. (1981). Acta Cryst. B37, 2030-2034. CrossRef details

Mayerle, J. J., Torrance, J. B. \& Crowley, J. I. (1979). Acta Cryst. B35, 2988-

2995. CrossRef details

Nonius (1998). COLLECT. Nonius BV, Delft, The Netherlands.

Otwinowski, Z. \& Minor, W. (1997). Methods in Enzymology, Vol. 276, Macromolecular

Crystallography, Part A, edited by C. W. Carter Jr \& R. M. Sweet, pp. 307-326. New York:

Academic Press.

Oxford Diffraction Ltd (2010). CrysAlis CCD, Version 1.171.33. Oxford Diffraction Ltd, Abingdon, Oxfordshire, England.

Sadohara, R. \& Matsuzaki, S. (1997). Phys. B, 239, 176-180. CrossRef CAS

Schomaker, V. \& Trueblood, K. N. (1998). Acta Cryst. B54, 507-514. Web of Science

CrossRef details

Sheldrick, G. M. (2008). Acta Cryst. A64, 112-122. CrossRef details

Tasaki, K., Matsuzaki, S. \& Yartsev, V. (1997). Solid State Commun. 103, 365-

369. CrossRef CAS

Torrance, J. B. \& Mayerle, J. J. (1981). Solid State Commun. 38, 1165-1169. CAS

Umland, T. C., Allie, S., Kulhlmann, T. \& Coppens, P. (1988). J. Phys. Chem. 92, 6456-

6460. CrossRef CAS

Wudl, F., Wobschall, D. \& Hufnagel, E. J. (1972). J. Am. Chem. Soc. 94, 670-

672. CrossRef CAS

Yamashita, Y., Tomura, M. \& I 